\title{
ON CERTAIN ENTIRE FUNCTIONS
}

I. M. SHEFFER

We shall say that an analytic function $f(z)$ has property $\mathcal{f}$ at a point $z_{1}$ if the sequence of derivatives $\left\{f^{(n)}\left(z_{1}\right)\right\}, n=0,1, \cdots$, takes on only a finite number of distinct values. An entire function of the form

$$
f(z)=Q(z)+\sum_{j=0}^{m-1} A_{i} \exp \left\{\omega^{j} z,\right.
$$

where $Q(z)$ is a polynomial and $\omega=\exp \{2 \pi i / m\}$, has property $\mathcal{F}$ at every point, but a function having property $\mathcal{F}$ at one point is not necessarily a special exponential sum (as we shall term a function of form (1)). We give three theorems whose conditions relate property $\mathcal{F}$ to special exponential sums.

THEOREM 1. If $f(z)$ has property $\mathcal{F}$ at two points, then it is a special exponential sum.

TheOREM 2. If $f(z)$ has property $\mathcal{F}$ at a point $z_{1}$, and if at a second point $z_{2} \neq z_{1}$ infinitely many derivatives are equal, then $f(z)$ is a special exponential sum.

Theorem 3. Let $r, \sigma, A$ be arbitrary positive numbers, with $r$ an integer and $\sigma \leqq 1$. Then there is an integer $V=V[r, \sigma, A]$ with the following property: Let $f(z)$ have property $\mathcal{F}$ at $z_{1}$ where the distinct values of $\left\{f^{(n)}\left(z_{1}\right)\right\}$ are $a_{1}, \cdots, a_{t}$, with $t \leqq r$ and

$$
\min \left|a_{i}-a_{j}\right| \geqq \sigma \cdot \max \left|a_{i}-a_{j}\right| \quad(i \neq j=1,2, \cdots, t) .
$$

If for a value $z_{2}$ in $0<\left|z_{1}-z_{2}\right| \leqq A$ there are more than $V$ equal quantities in the sequence $\left\{f^{(n)}\left(z_{2}\right)\right\}$, then $f(z)$ is a special exponential sum.

It is clear that Theorem 3 implies 2 and 2 implies 1 , so it suffices to establish 3. Since a translation in the independent variable does not alter the essential conditions, we may suppose that $z_{1}=0$ and (changing the letter) that $z_{2}=a$. Then

$$
f(z)=\sum_{0}^{\infty} c_{n} \frac{z^{n}}{n !}=\sum_{0}^{\infty} d_{n} \frac{(z-a)^{n}}{n !}
$$

where $c_{n}=f^{(n)}(0)$ and $d_{n}=f^{(n)}(a)$. If we differentiate (3) $n$ times and set $z=a$, we obtain the linear relations

Presented to the Society, April 26, 1952; received by the editors November 30, 1951. 


$$
c_{n}+\frac{a}{1 !} c_{n+1}+\frac{a^{2}}{2 !} c_{n+2}+\cdots=d_{n} \quad(n=0,1,2, \cdots)
$$

Define

$$
\delta_{k}(A)=\sum_{p=1}^{\infty} \frac{A^{p}}{(k+1)(k+2) \cdots(k+p)} .
$$

Since $\delta_{k}(A) \rightarrow 0$ as $k \rightarrow \infty$, there is a smallest value $k=K=K[\sigma, A]$ such that

$$
\delta_{K}(A)<\sigma .
$$

We shall show that a possible choice of $V$ is $V=r^{K}$. Set

$$
P_{n, 8}(a)=c_{n}+\frac{a}{1 !} c_{n+1}+\cdots+\frac{a^{s}}{s !} c_{n+8} .
$$

Then

$$
\begin{aligned}
d_{n}-d_{q} & =\left\{P_{n, K-1}(a)-P_{q, K-1}(a)\right\} \\
+ & \frac{a^{K}}{K !}\left\{\left(c_{n+K}-c_{q+K}\right)+\sum_{s=K+1}^{\infty}\left(c_{n+s}-c_{q+8}\right) \frac{a^{s-K}}{(K+1) \cdots s}\right\} .
\end{aligned}
$$

Since $\left|c_{n+s}-c_{q+8}\right| \leqq \max \left|a_{i}-a_{j}\right|$, and $|a| \leqq A$, the last sum in (8) cannot exceed in magnitude the quantity $\delta_{K}(A) \cdot \max \left|a_{i}-a_{j}\right|$.

Now suppose $d_{n_{1}}=d_{n_{2}}=\cdots=d_{n_{v}}$, where $v>r^{K}$. Since each $c_{j}$ has one of the values $a_{1}, \cdots, a_{t}$, therefore for fixed $s$ there are at most $r^{o+1}$ different expressions $P_{n, 8}(a)$. Hence of the expressions $P_{n_{j}, K-1}(a)$, $j=1, \cdots, v$, at least two are equal; say for $n=n_{\alpha}, n_{\beta}$. Taking $n=n_{\alpha}$, $q=n_{\beta}$ in (8), we see that

$$
\text { (9) } c_{n_{\alpha+K}}-c_{n \beta+K}=-\sum_{s=K+1}^{\infty}\left(c_{n_{\alpha+8}}-c_{n \beta+8}\right) \frac{a^{s-K}}{(K+1) \cdots s} \text {; }
$$

so

$$
\left|c_{n_{\alpha}+K}-c_{n \beta+K}\right| \leqq \delta_{K}(A) \cdot \max \left|a_{i}-a_{j}\right|<\sigma \cdot \max \left|a_{i}-a_{j}\right| .
$$

This is in contradiction to (2) unless $c_{n_{\alpha}+K}=c_{n_{\beta}+K}$. We may therefore rewrite (9) as

$$
\left(c_{n_{\alpha}+K+1}-c_{n_{\beta}+K+1}\right)=-\sum_{s=K+2}^{\infty}\left(c_{n_{\alpha}+s}-c_{n \beta+\delta}\right) \frac{a^{s-K-1}}{(K+2) \cdots s},
$$

and from this conclude that $c_{n_{\alpha}+K+1}=c_{n_{\beta}+K+1}$, and so on, with the result that 


$$
c_{n_{\alpha}+K+j}=c_{n \beta+K+j}, \quad j=0,1,2, \cdots .
$$

Thus, beginning at least with the index $n=n_{\alpha}+K$, the sequence $\left\{c_{n}\right\}$ is periodic; and it is an easy consequence that $f(z)$ is a special exponential sum.

REMARKS. (i) A theorem ${ }^{1}$ of Szegö states that if the coefficients $\left\{c_{n}\right\}$ of the series $F(z)=\sum_{0}^{\infty} c_{n} z^{n}$ take on only a finite number of distinct values, then either (a) $F(z)$ has the circle $|z|=1$ as cut, or (b) $F(z)$ is a rational function of the form $F(z)=P(z) /\left(1-z^{m}\right)$ where $m$ is a positive integer and $P(z)$ is a polynomial. Both cases arise, and this suggests the problem of assigning a further condition to insure (let us say) that case (b) holds. If we introduce the entire function $f(z)$ $=\sum_{0}^{\infty} c_{n} z^{n} / n$ ! associated with $F(z)$, then $f(z)$ has property $\mathcal{F}$ at $z=0$; and case (b) is easily seen to be equivalent to the condition that $f(z)$ be a special exponential sum. Thus, the conditions of any one of Theorems 1, 2, 3 suffice to guarantee case (b).

(ii) Theorem 1 shows that relative to property $\mathcal{f}$ there are only three possibilities for an analytic function $f(z)$ : either it has property $\mathcal{F}$ at no point whatever, or at just one point, or at all points. Also, as noted by the referee, Theorem 1 can be formulated in this way: If an entire function of exponential type has for its indicator diagram a circle, center at the origin, then it cannot have property $\mathcal{f}$ at two points.

(iii) In the course of the proof of Theorem 3 it was shown that a permissible choice of $V$ is $V=V[r, \sigma, A]=r^{K}$, where $K=K[\sigma, A]$. It would be of interest to determine, for given $r, \sigma, A$, the smallest possible $V$. It is conceivable that this minimum $V$ is independent of one or more of the quantities $r, \sigma, A$.

The Pennsylvania State College

${ }^{1} \mathrm{G}$. Szegö, Über Potenzreihen mit endlich vielen verschiedenen Koeffizienten, Berl. Ber. (1922) pp. 88-91. A proof is also found in P. Dienes, The Taylor series, Oxford, 1931, pp. 324-327. 